\title{
RANGE-INDEPENDENT SEGMENTATION OF SIDESCAN SONAR IMAGES WITH UNSUPERVISED SOFM ALGORITHM (SELF-ORGANIZING FEATURE MAPS)
}

\author{
A. Nait-Chabane Lab-STICC UMR CNRS 6285, ENSTA Bretagne, Brest, Fr. \\ B. Zerr Lab-STICC UMR CNRS 6285, ENSTA Bretagne, Brest, Fr. \\ G. Le Chenadec Lab-STICC UMR CNRS 6285, ENSTA Bretagne, Brest, Fr.
}

\section{INTRODUCTION}

The sidescan sonar records the energy of an emitted acoustical wave backscattered by the seafloor, orthogonally to the track followed. The statistical properties of the backscattered energy change with the nature of the seafloor, which allows for a segmentation of the seabed into homogeneous regions. However, the statistical description of the backscattering is not constant over the full swath of the sonar [1], due to the propagation of acoustic wave in water and characteristics of sonar system beam (directivity, duration of emitted signal, insonified area, etc.) [2]. Thus, the segmentation results of algorithms applied to sidescan sonar images are non optimal.

As with most other digital remote sensing images data, the preprocessing of sidescan sonar images includes two steps detailed in [3]: geometric corrections and radiometric corrections. Some of the geometric problems that need to be corrected for sidescan sonar images are: the water column offset, slant-range to ground-range projection, distortion due to the resolution ratio between the along and across-direction and change of ship's velocity. The second preprocessing step is radiometric corrections. Typically, most algorithms used for radiometric corrections are: shading correction due to the energy decreasing from near to far-range, correction for speckle.

In the literature, several works have studied the effect of variability of seabed backscattering response on image segmentation results. In [1], the data are first corrected for artifacts related to the wave propagation and characteristics of the sonar system. This approach requires strong knowledge of the system and the conditions of acquisition. Further work, [4] use a preprocessing step in which the data are corrected by estimating range-dependent variations. In [5], correction model for compensation of sonar sidescan images is proposed using three multiplicative corrections factors. These factors describe changes in the sonar altitude and angular dependencies, mainly due to the vertical beam pattern and grazing angle.

Seafloor segmentation approaches could be categorized as either parametric or non-parametric. The parametric ones are based on modeling the probability distribution of the backscattering energy. These models take into account the conditions of acquisition, the properties of the seabed and the angular variations of signal backscattering. The most used models include the Rayleigh model, Weibull and $K$ distributions. The parametric models are more often used in Bayesian framework , [6][7]. In [7], a measure of similarity between the distributions of the different types of seabed sediments is estimated. The measure used is a weighted sum of the Kullback-Leiber divergence. The weight is introduced in the estimation of the measure that considers the influence of incidence angle. The nonparametric approaches do not consider the conditions of acquisition and physical properties of the backscattering signal. These approaches consider seabed backscatter as textured image. Most features commonly used are Haralick attributes based on the co-occurrence matrix [8], spectral analysis (Fourier transform) [9] and time scale analysis (wavelet) [10].

The statistical description of the backscattering is not constant over the full swath of the sonar. Making the backscattering energy independent of the grazing angle is a more difficult challenge, conventionally solved by considering a flat seabed and by using either Lambert's law or an empirical law estimated from the sonar data. To avoid the definition of a physical law describing the change in energy with grazing angle, the proposed algorithm divides the slant range into small stripes, where the statistics can be considered unaltered by the grazing angle variations. The starting stripe at mid sonar slant 
range is segmented with an unsupervised classifier. Then, from the knowledge acquired on the segmentation of this first stripe, the classifier adapts its segmentation to the neighboring stripes, allowing slight changes of statistics from one stripe to the other. The operation is repeated until the beginning and the end of the slant range are reached. The proposed approach for unsupervised segmentation of a slant range stripe is an adaptation of the Kohonen algorithm SOFM (Self-Organizing Feature Maps) [11]. This algorithm is a competitive neural network based on the biological functioning of the cerebral cortex. SOFM algorithm is different from other artificial neural networks that it uses a neighborhood function to preserve the topological properties of the input space. The algorithm is applied to raw sonar images and does not require a priori knowledge about the data. The paper is organized as follows. In section 2, the dependence of the backscattering to grazing angle and proposed process of splitting of sonar images are discussed. Then, the principle of the proposed algorithm is given in section 3. In section 4, the different texture features used to discriminate the seabed are detailed. In section 5, a brief review of the learning process of SOFM algorithm is gived . Section 6 provides experimental results of the proposed algorithm on real sonar images and compared its performance with the same SOFM algorithm applied on sonar images a priori corrected then compared with results obtained with K-means algorithm. Finally, we conclude with remarks and some perspectives in section 7 .

\section{RANGE DEPENDENCE}

In all sonar systems at the receiving processor output, the amplitude of echo will be function of sonartarget distance. To compensate these variations, a common solution is to correct the signal received in domain time, using a physical or empirical law [12]. The energy loss due to propagation of acoustic waves in the marine environment has a dual origin, the first loss is the geometric divergence and a second loss due to absorption corresponding to a conversion of acoustic energy into heat dissipation. Reverberation is one of the most important physical phenomena in sonar. It is characterized by a surfacic or volumic index which is a function of the pulse duration, the directivity of the antenna the level of the transmitted signal, the texture of the substance but also the grazing angle [12]. Several empirical models give the values of the reverberation index as a function of grazing angle and type of bottom, for example the Lambert's model gived in figure 1.

$$
R F(\alpha)=B S_{0}+20 \log (\sin (\alpha))
$$

$\alpha$ :Grazing angle $\left(^{\circ}\right), B S_{0}:$ constant $(d B)$.

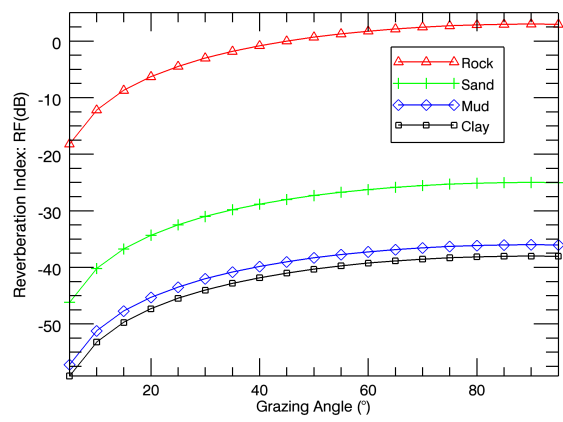

Figure 1: Reverberation Index as function of grazing angle and type of bottoom (Lambert's model)

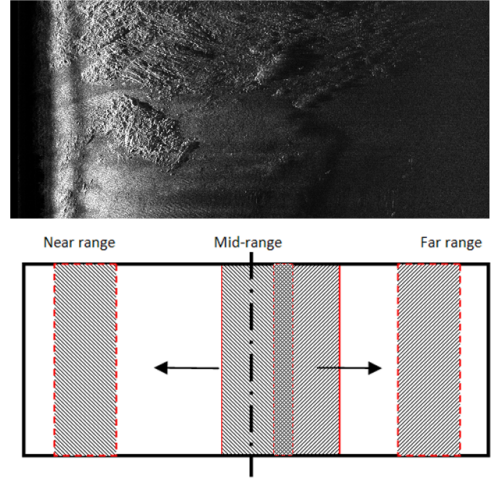

Figure 2: Splitting process

\subsection{SPLITTING PROCESS OF SONAR IMAGE}

Making energy backscattered from seafloor independent of the grazing angle is a probelm conventionally solved by considering a flat seabed and using either Lambert's law as shown on figure 1 or 
empirical law. To avoid the definition of a physical law describing the dependence of the energy with the range of the sonar, we propose to divide the range into small overlapping stripes. In a given stripe, the calculated statistics can be considered unaltered by the range. At first, the central stripe representing the mid-range sonar is segmented with an unsupervised classifier. Then, from the knowledge on the segmentation of the first stripe, classification results are propagated and adapted to its neighbors, until both edges of the image are reached. We schematize this process in figure 2.

\section{PROPOSED ALGORITHM}

The algorithm used is unsupervised segmentation based on SOFM (Self-Organizing feaure maps) Kohonen neuronal network [11]. Several methods of texture analysis are proposed in the literature, there are methods based on first order statistics, other based on second order(co-occurrences matrice) and methods based on spectral analysis [13]. In our case, the features derived from texture analysis combine two techniques. The first technique of texutre analysis is based on the estimation of a co-occurrences matrices and the different Haralick features derived from. The ability of these attributes to discriminate textures seabed has been shown in several work [14], [15]. The second technique analysis is the spectral analysis. The input data (vector attributes) of the SOFM result from a texture analysis of the sonar image. The proposed algorithm consists of five stages:

1. Splitting the raw image into stripes,

2. Creation of vector attributes using texture analysis,

3. Reducing the dimensionality of the vector attributes using the SOFM algorithm,

4. Segmentation of the input image into disjoint classes by analyzing the topology map created by the SOFM,

5. Coding of the neuron map using colors.

\section{FEATURES EXTRACTION}

\subsection{TEXTURAL FEATURES}

The Grey Level Co-occurrence Matrix (GLCM) is a second-order statistical tool used for texture analysis of images proposed by Haralick [16]. It has been applied successfully on satellite images [17] and medical images [18], etc. The GLCM of an image $I$ of size $N \times M$ coded in $N g$ grayscale is a matrix of size $N g \times N g$. It is obtained by calculating the number of transitions for each pair of gray level $(i, j)$ of a given distance $d$ and angular direction $\theta$. Analytical expression of GLCM on an image $I$ is given by:

$$
\operatorname{GLCM}_{(d, \theta)}(i, j)=\sum_{i_{1}=1}^{n} \sum_{i_{1}=1}^{m}\{I(n, m)=i, I(n+d \cos \theta, m+d \sin \theta)=j\}
$$

$(n, m) \in[1, N] \mathrm{X}[1, M]$. The GLCM is calculated in 4 directions to describe the texture content: horizontal $\left(d=1,0^{\circ}\right)$, vertical $\left(d=1,90^{\circ}\right)$, right- $\left(d=1,45^{\circ}\right)$ and left-diagonal $\left(d=1,135^{\circ}\right)$ directions. Haralick [19] proposed a set of 14 local features. The different Haralick parameters are calculated from the normalized $G L C M$.

In our work the following Haralick features are used : Entropy, Contrast, Heterogeneity, Homogeneity, Correlation, Maximum of probability, Kurtosis and Elongation Factor. 


\subsection{SPECTRAL FEATURES}

Two-dimensional transforms have been used extensively in image processing to tackle problems such as image description and enhancement. Of these, the Fourier transform is one of the most widely used [20]. Fourier analysis can be used to study the properties of textured scenes, for example the power spectrum reveals information on the coarseness/fineness (periodicity) and directionality of a texture. Texture directionality is preserved in the power spectrum because it allows directional and non-directional components of the texture to be distinguished [21]. These observations have given rise to two powerful approaches for extracting texture primitives from the Fourier power spectrum, namely, ring filters.

In our case, six attributes computed from the Fourier transform. Three attributes calculated directly from the magnitude spectrum of the Fourier transform $F(u, v)$, are: the mean, the variance and the power of the magnitude.

The other three parameters are calculated from frequency filtering based on the Fourier Transform. Circular filters applied in three spectral bands:low, medium and high frequencies. The operator takes an image and a filter function in the Fourier domain. This image is then multiplied with the filter function $H(u, v)$ in a pixel-by-pixel. Such us, $\mathrm{F}(\mathrm{u}, \mathrm{v})$ is the input image in the Fourier domain, $\mathrm{H}(\mathrm{u}, \mathrm{v})$ the filter function and $G(u, v)$ is the filtered image. The filters used in our work are ideal filters for lowpass, bandpass and highpass. For example, the bandpass filter $H_{L F}(u, v)$ leaves all frequencies unchanged between the cut-off $D_{L F}$ and $D_{H F}$ frequencies and suppresses other frequencies.

$$
H_{M F}(u, v)= \begin{cases}1 & \text { if } \mathrm{D}_{L F}<\sqrt{u^{2}+v^{2}}<\mathrm{D}_{H F} \\ 0 & \text { if } \mathrm{D}_{H F}<\sqrt{u^{2}+v^{2}}<\mathrm{D}_{H F}\end{cases}
$$

$D_{L F}$ is the low cut-off frequency and $D_{H F}$ is the high cut-off frequency.

Low frequency power (LFP)

$$
\begin{gathered}
G_{L F}(u, v)=H_{L F}(u, v) \cdot F(u, v) \\
L F P=\frac{\frac{1}{M N} \sum_{x=0}^{M-1} \sum_{u=0}^{N-1}\left(G_{L F}(u, v)^{2}\right)}{E}
\end{gathered}
$$

where $\mathrm{F}(\mathrm{u}, \mathrm{v})$ is the input image in the Fourier domain, $H_{L F}(u, v)$ the low filter mask and $G_{L F}(u, v)$ is the low filtered image normlized by the power spectrum $\mathrm{E}$.

Medium frequency power (MFP)

$$
\begin{gathered}
G_{M F}(u, v)=H_{M F}(u, v) \cdot F(u, v) \\
M F P=\frac{\frac{1}{M N} \sum_{u=0}^{M-1} \sum_{v=0}^{N-1}\left(G_{M F}(u, v)^{2}\right)}{E-L F P}
\end{gathered}
$$

$H_{M F}(u, v)$ the medium filter mask, $G_{M F}(u, v)$ is the medium filtered image normlized only with power spectrum containde in the medium and high frequency(E-LFP).

High frequency power(HFP)

$$
H F P=1-[L F P+M F P]
$$

An example of the filtering band on three types of texture (rock,ripples and sand) is shown on Figure 3.

\subsection{FEATURES VECTOR}

The sidescan sonar raw images are divided into vertical stripes of 96 pixels width. On each stripe, 39 Features are calculated : 32 features of Haralick and six(6) Fourier features. The Haralick parameters are calculated using an analysis window of $96 \times 32$ pixels, with a step of 32 pixels along range and 8 pixels along track. To take into consideration sonar altitude variations, sonar altitude is inserted as $39^{\text {th }}$ feature. 


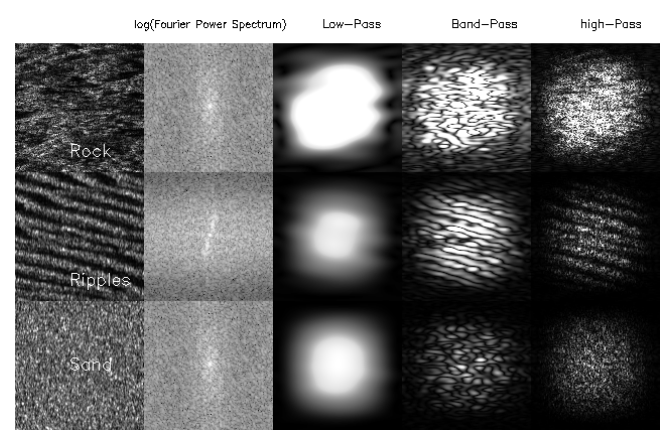

Figure 3: Circular filtring

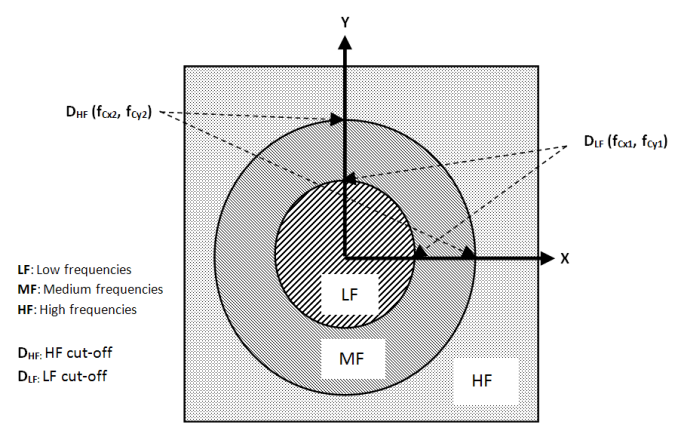

Figure 4: Representation of filter gabarit

\section{UNSUPERVISED SEGMENTATION}

The purpose of unsupervised learning methods is to develop an optimal partitionning, i.e clustring, of the data set to be analyzed. Cluster analysis is the organization of collection of patterns wich are usually represented as vectors of measurments, into clusters based on similarities. Approaches to unsupervised learning include clustering (K-means, mixture models, hierarchical clustering, selforganizing feature map (SOFM), etc).

\subsection{K-MEANS CLUSTERING}

K-means is an unsupervised learning algorithm, its purpose is to divide observations into $\mathrm{k}$ partitions or clusters in which each observation belongs to the partition with the nearest average. In this work, principal components analysis (PCA) is used to reduce the dimensionality of feature vector space of each stripe before application of K-means clustering. The PCA"gives orthogonal linear combination ordered by amount of variance they present. Typically more than $90 \%$ of the variance contained in the first three components. So,to facilitate human interpretation and further processing, it convenient to reduce the dimensionality of feature vector space from 39 to only 3 components. An euclidean metric is not well suited for acoustic records after PCA because the three PCA axes represent distinctly different amount of variance and thus should not be treated equally [15]. So, Mahalanobis metric is used for K-means clustering. This metric obtained with normalization by variance of euclidean metric in each PCA direction.

\subsection{K-MEANS WITH CONFUSION MATRIX (KM-CM)}

We performed the same process of splitting stripes of sonar image for K-means algorithm segmentation. The number of clusters initialized for $\mathrm{K}$-means is $K=4$. The problem encountered is the transition management and continuity of classes between the stripes of image. This problem is due by the random initilaization of K-means clusters applied separately on each stripe. To overcome this problem, inter-stripes management is performed by confusion matrices computed on the overlapping pixels of consecutive stripes, calling this process K-means with confusion matrix (KM-CM). The result of segmentation by KM-CM algorithm is shown in figure 9

\subsection{SEGMENTATION SOFM ALGORITHM}

In the study of brain function, biologists have found that activities such as vision, speech and hearing are associated with specific areas of the cortex cerebral. These surfaces are arranged so as to preserve the topology of sensorial sensors. For example, Hubel and Weisel (1947) [22] have shown that two near areas in the visual cortex correspond to two near areas in the retina. This organization inspires Kohonen to develop Self-organizing feature maps (SOFM) algorithm [11]. SOFM transforms 


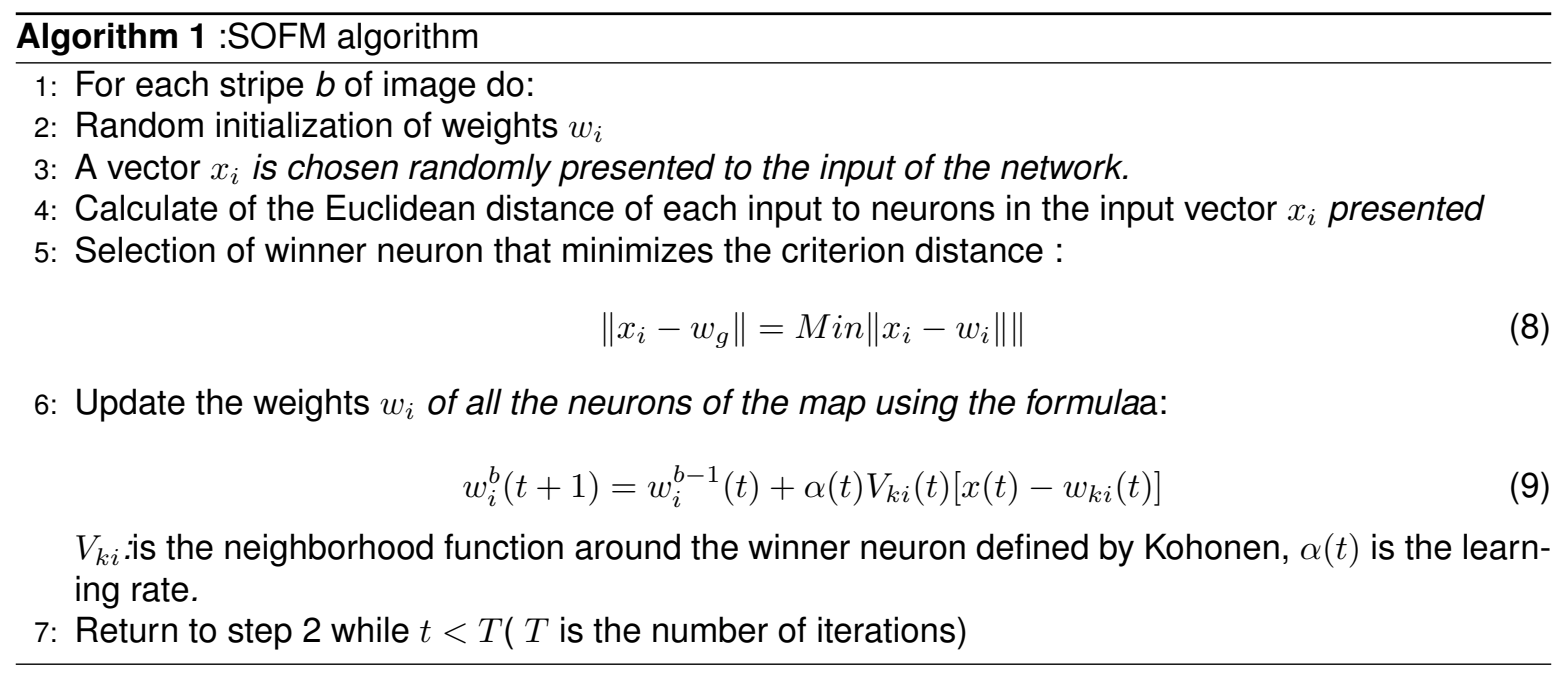

the input of high dimension into a one or two dimensional discrete map subject to a topological (neighborhood preserving) constraint. SOFM algorithm allows reduction of dimensionality of feature vector space. So in this case, we do not need to use PCA for reduction dimensionality and euclidean metric is used for clustering. Our tests are performed of classification for $2 \times 2$ Kohonen map size. A Kohonen map represents $m^{2}$ different classes, that is to say a $2 \times 2$ card presents 4 classes. The SOFM algorithm preserves the topology, so we use a color space suitable for the topology, two neurons with similar weights (in the feature space) are represented with similar colors.

\subsubsection{Learning phase}

The SOFM algorithm is a type of competitive artificial neural network generally consists of two layers of neurons. The first layer is used only to present the observations or vectors $x_{i}^{p}$ of the input space. The second is the layer of competition , the geometry of this network is defined a priori. Each element of the input layer is connected to all neurons of the second layer to allow the self-organization. The link between the two layers is made by vectors denoted $w_{i}^{p}$ (synaptic weights). These weights are updated iteratively by the learning algorithm based on the neighborhood. The principle of learning of the SOFM algorithm is to promote the winner neuron by comparing the input vector to which it owes its victory. The learning phase is to update the weights $\left(w_{i}\right)$ in such a way that vectors close in distance and topology in the input space are associated with nearby neurons on the map. In our case, we note that the learning of a given vertical band of the image depends on the learning of the previous band, which allows us to keep the continuity of classes from one band to another. The SOFM model chosen in our application is a grid of $m \times m$ neurons $(m=2,3,5 \ldots)$. Each neuron $n_{i}$ is connected to an input $x_{i}^{p} \in R^{p}$ by a vector of weight $w_{i}=\left(w_{i 1}, w_{i 2}, \ldots, w_{i p}\right) \in R^{p}$. The main phases of the SOFM algorithm are gived on Algorithm 1.

The generalization ability of the SOFM algorithm depends on two parameters: the learning rate $\alpha(t)$ and neighborhood function $V_{k i}(t)$. These two parameters are chosen heuristically [11]. The neighborhood function described in step 6 of the algorithm 1 has the form of a Gaussian function gived by:

$$
V_{k i}(t)=\exp \left[-\frac{d^{2(k, i)}}{2 \sigma^{2}(t)}\right]
$$

$\sigma(t)$ the width of the neighborhood function at iteration $t$.

$d(k, i)=|k-i|$ euclidean distance between neuron $k$ and vector $i$ According to Kohonen [11] the convergence of the learning process requires that the neighborhood function $V_{k i}(t) \rightarrow 0$ as $t \rightarrow T(T$ : number of iterations of the process). To ensure convergence of process, the learning rate $\alpha(t)$ and standard deviation $\sigma(t)$ of the neighborhood function should be two monotone decreasing functions. 


$$
\sigma(t)=\sigma(0)\left[\frac{\sigma(T-1)}{\sigma(0)}\right]^{\left(\frac{t}{T-1}\right)}
$$

$\sigma(0)$ : Half of the initial number of neural network.

$\sigma(T-1)=1 / 2$

$$
\alpha(t)=\alpha(0)\left(\frac{1-t}{T}\right)
$$

$\alpha(0)=0.03$ and $T$ : numbers of iterations of the process (25000)

\section{EXPERIMENTAL RESULTS}

The sonar images used for our study are provided by the GESMA (Group d'Etudes Sous-Marines d'Atlantique). Data were acquired by the Klein 5000 sidescan sonar at the BP02 campaign, recorded between May and June 2002 on the Cinque Terre region and Framura in Italy. The experiment is conducted by NATO/NURC. The frequency of sonar is $455 \mathrm{kHz}$. For low-resolution, maximum range is $150 \mathrm{~m}$ on each side of sonar which gives a swath of $300 \mathrm{~m}$. In high resolution a range is $75 \mathrm{~m}$ so a swath of $150 \mathrm{~m}$. In our study we use high resolution images (swath of $150 \mathrm{~m}$ ). The along-direction resolution is $10 \mathrm{~cm}$ and across-direction resolution is $3 \mathrm{~cm}$.

To assess the performances of our new approach, two comparisons are made. First, we compare the segmentation results obtained by application of $\mathrm{KM}-\mathrm{CM}$ algorithm on raw sonar data with performances of proposed algorithm. Then a comparison between results of proposed algorithm with segmentation results of sonar images previously corrected then segmented with SOFM algorithm applied to the sonar images without splitting process. The corrections applied are geometric and radiometric. Geometric corrections consist on the water column offset and slant range to ground range projection. For the radiometric correction, a compensation of the variation of backscattering energy by normalization of amplitudes is made. The result of sonar image corrected and segmented is shown respectively in figure 5 and figure 6 . The segmentation result of KM-CM clustering algorithm with number of clusters $k=4$ is given in figure 9 and segmentation result of proposed algorithm is given in figure7. To study the quantitative performance of the proposed algorithm, a confusion matrix is calculated on the overlap of two adjacent stripes. The calculation is done for each side of the image port and starboard. The results of the confusion matrix of the result obtained by the KM-CM algorithm presented in figure 9 and the proposed algorithm in figure 7 are represented on the table 10. Clearly, we show that proposed algorithm gives better result than KM-CM clustering and manages the continuity of classes between adjacent stripes of image.

The last comparison considers an homogeneous sandy area. On such area, unsupervised classification should give a unique class. The KM-CM class algorithm gives the worst result with $54 \%$ of the even correctly classification. The SOFM applied on the preprocessed data (geometric and radiometric corrections) shows better results as $85 \%$ of area belongs to the major class. Proposed algorithm applied on the divided image shows that the homogenous area as almost represented by a unique class $99.97 \%$. The results of segmentation by different algorithms for homogeneous sandy area are given in figure 11 and quantitative representation of rate of pixels of each class is gived in figure 12 .

\section{CONCLUSION}

In this paper, an unsupervised algorithm for segmentation of raw sidescan sonar images is proposed. This algorithm is based on the adaptation of the Kohonen SOFM algorithm. Segmentation performances of the proposed algorithm are compared with those obtained by the KM-CM algorithm using confusion matrix. Another comparison with a sandy homogeneous area is made. The proposed approach allows a better classification rate and does not depend on the incidence angle of the sonar. The proposed algorithm performs a segmentation independent to the grazing angle, without a priori 
information on the dependence on the incidence angle of the system acquisition (i.e. without image preprocessing) by managing transitions and continuity of classes between the adjacent stripes. Further work will address the use of a dynamic map (i.e. size of the SOFM adapting to the complexity of the segmentation task) and the improvement of the color coding of the map for a better representation of the physical nature of the sediment.

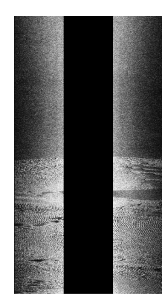

Figure 5: Sonar image

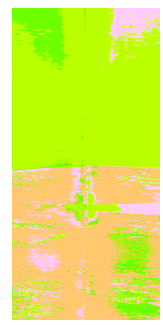

Figure 8: SOFM of preprocessed image.

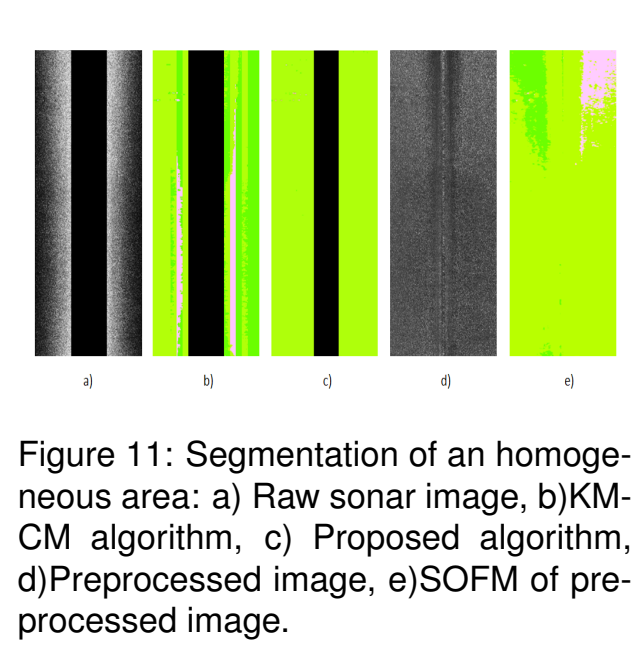

Figure 11: Segmentation of an homogeneous area: a) Raw sonar image, b)KMCM algorithm, c) Proposed algorithm, d)Preprocessed image, e)SOFM of preprocessed image.

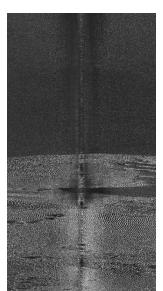

Figure 6: Preprocessed image.

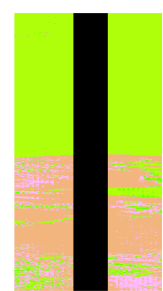

Figure 7: Proposed algorithm.

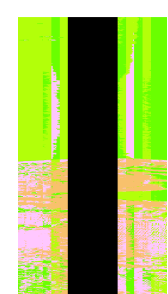

Figure 9: KM-CM algorithm. gorithm.

\begin{tabular}{|c|c|c|c|}
\hline \multicolumn{4}{|c|}{ Rate of good classification (\%) } \\
\hline \hline \multicolumn{3}{|c|}{ KM-CM } & Propo.algorithm \\
\hline \hline Port & Starboard & Port & Starboard \\
\hline 6 & 42 & 86 & 85 \\
\hline 46 & 42 & 78 & 63 \\
\hline 79 & 35 & 74 & 80 \\
\hline 53 & 39 & 71 & 80 \\
\hline 56 & 35 & 76 & 79 \\
\hline 70 & 40 & 60 & 70 \\
\hline
\end{tabular}

Figure 10: Rate of good classfication:KM-CM,Proposed al-

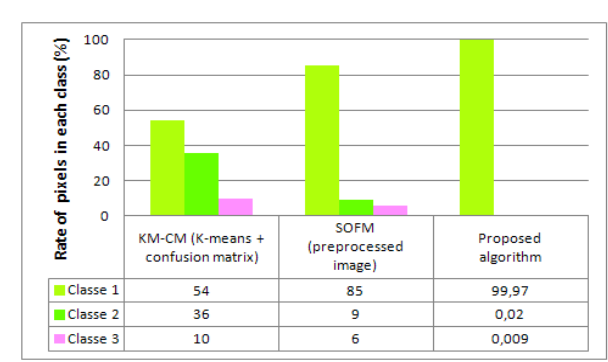

Figure 12: Comparison of segmentation of an homogeneous area by the three algorithms: KM-CM, Proposed algorithm, SOFM of preprocessed image.

\section{ACKNOWLEDGEMENTS}

The authors would like to thank GESMA (Groupe d'Etudes Sous-Marines d'Atlantique)and the NATO Undersea Research Centre (NURC) for providing the sidescan sonar images. 


\section{REFERENCES}

1. G. L. Chenadec and J.-M. Boucher. Sonar image segmentation using the angular dependence of backscattering distributions. In Proceeding ocean, pp. 147-152, 2005.

2. L. Hellequin, J.-M. Boucher, and X. Lurton. Processing of high-frequency multibeam echo sounder data for seafloor characterization. IEEE Journal of Oceanic Engineering, 28(1): pp. 78-89, 2003.

3. J. Chavez, P.S, J. Isbrecht, P.Galanis, G. Gabel, S. Sides, D. Soltesz, S. Ross, and M. Velesco. Processing, mosaicking and management of the monterey bay digital sidescan-sonar images. Marine Geology, 181(1-3): pp. 305-315, 2002.

4. S. Anstee. Removal of range-dependent artifacts from sidescan sonar imagery. Tech. report, Technical Report DSTO-TN-0354, DSTO Aeronautical and Maritime Research Laboratory, Australia, 2001.

5. C. Capus, A. Banks, E.Coiras, I. Ruiz, C. Smith, and Y. Petillot. Data correction for visualisation and classfication of sidescan sonar imagery. iet-rsn, 2(3): pp. 155-169, Jun 2008.

6. G. L. Chenadec, J.-M. Boucher, X. Lurton, and J.-M. Augustin. Angular dependence of statistical distributions for backscattered signals: modelling and application to multibeam echsounder data. In Ocean, vol. 2, pp. 897-903, 2003.

7. I. Karoui, R. Fablet, J.-M. Boucher, and J.-M. Augustin. Seabed segmentation using optimized statistics of sonar textures. IEEE Journal of Oceanic Engineering, 47(3): pp. 1621-1631, Jun 2009.

8. M. Lianantonakis and Y. Petillot. Sidescan sonar segmentation using texture descriptors and active contours. IEEE Journal of Oceanic Engineering, 32(3): pp. 744-752, Jul 2007.

9. T. Hsu, A. Calway, and R. Wilson. Texture analysis using the multiresolution fourier transforms. In In Proc. 8th scandinavian Conference on image Analysis, pp. 823-830, 1993.

10. T. Celik and T. Tjahjadi. A novel method for sidescan sonar image segmentation. IEEE Journal of Oceanic Engineering, 36(2): pp. 186 - 194, Apr 2011.

11. T. Kohonen. Self-organizing formation of topologically correct feature maps. In Biological $\mathrm{Cy}$ bernitics, vol. 46, pp. 59-69, 1982.

12. X. Lurton. An Introduction to Underwater Acoustics: principles and applications. springer, 2002.

13. F. Tomita and S. Tsuji. Computer analysis of visual textures. Kluwer Academic Publishers, 1990.

14. N. Pican, E. Trucco, M. Ross, D. Lane, Y. Petillot, , and I. T. Ruiz. Texture analysis for seabed classification: Co-occurrence matrices vs self-organizing maps. In OCEAN'98 Conference Proceeding, 1998.

15. J. Preston. Automated acoustic seabed classification of multibeam images of stanton banks. Applied Acoustics, 70(10): pp. 1277-1287, 2009.

16. R. Haralick. Statistical and structural approaches to texture. In Proceeding IEEE, pp. 786-804, 1979.

17. D. Marceau, P. Howarth, J. Dubois, and D. Gratton. Evaluation of the grey-level coocurence matrix method for land-cover classifcation using spot imagery. IEEE on Geoscience and Remote Sensing, 28(4): pp. 513-519, Jul 1990.

18. D. Sercic and S. Loncaric. Enhancement of mammographic for detection of microcalcifications. In Proceedings of the IX European Signal Processing Conference ,Island of Rhodos, Greece, vol. 2, pp. 693-696, Sep 1998. 
Proceedings of the 11th European Conference on Underwater Acoustics

19. R. Haralick. Textural features for image classification. IEEE Trans, Systems, Man and Cybernetics, SMC-3, 6: pp. 610-621, 1973.

20. X. Tang and K. Stewart. Texture classification using wavelet packet and fourier transforms. In Ocean'95, vol. 1, p. 387, 1995.

21. R. Bajcsy. Computer description of textured surfaces. In Proceedings of the Joint Conference on Artificial Intelligence, pp. 572-579, Aug 1973.

22. T. Wiesel and D. Hubel. Single-cell responses in striate cortex of kittens deprived of vision in one eye. Journal of Neurophysiology, 26: pp. 1003-1007, 1963. 